\title{
The Synthesis of the Reference Image and Algorithms for Vehicle Navigation Systems
}

\author{
Oleksandr Tymochko', Andrii Ttystan ${ }^{2}$, VladyslavUshan $^{3}$, NataliiaYeromina ${ }^{4}$, OlehDmitriiev $^{5}$, Volodymyr \\ Mazharov $^{6}$, Ivan Padalka ${ }^{7}$, Iryna Hannoshyna ${ }^{8}$, Igor Masik', Andrii Zazirnyi ${ }^{10}$ \\ ${ }^{1}$ Department of Air Navigation and Combat Control of Aviation Ivan KozhedubKharkiv National Air Force \\ University, Kharkiv, Ukraine, timochko.alex@gmail.com \\ ${ }^{2}$ Department for the Development of Air Force Groups at the Air Force Scientific Center Ivan KozhedubKharkiv \\ National Air Force University, Kharkiv, Ukraine, andr.tristan@gmail.com \\ ${ }^{3}$ Department of Aviation Tactics Ivan KozhedubKharkiv National Air Force University, Kharkiv, Ukraine, \\ pelsh1976@ukr.net \\ ${ }^{4}$ Department of Electronic Computers, Kharkiv National University of Radio Electronics, Ukraine, Kharkiv, \\ nataliia.yeromina@nure.ua \\ ${ }^{5,6,7}$ Department of Flight Operations, Aerodynamics and Flight DynamicsFlight Academy of National Aviation \\ University, Kropyvnytskyi, Ukraine, Dmitronik70@i.ua \\ ${ }^{8,9,10}$ Department of Navigation and Ship's Handling, State University of Infrastructure and Technologies, Kyiv, \\ Ukraine,iramsu@meta.ua
}

\begin{abstract}
The presented results of the synthesis of the optimal reference image (RI) and the algorithms for the vehicle navigation systems were acquired based on the tracking and comparison method. The synthesis of the optimal RI, corresponding to a stable component of an informative parameter is based on the use of the concepts of the scale of measurement theory. It has been suggested to formalize the brightness component of the RI, as well as shifts, rotations of the RI with respect to the current image $(\mathrm{CI})$, as well as an operation of the enumeration of CI fragments having the matching dimensions of the RI in terms of the Granander Image Theory.Summary ratios for describing models of current and reference images and optimal algorithms for forming corrective signals have been obtained.
\end{abstract}

Key words: Vehicles, navigation systems, invariant informative parameters, optimal reference image, optimal algorithms.

\section{INTRODUCTION}

In the implementation of high-precision air-, sea- and land-launched vehicle navigation, autonomous systems are widely used as corrective systems; they are based on the determination of navigation parameters in accordance with the information about different geophysical fields and the further use of the measurement results of these parameters for the formation of correction signals of the main navigation system [1, 2]. The determination of the correction signals is based on a comparison of the CI, which can be characterized by the distribution of the informative parameter of the respective physical field in the area of vision of the system, with one or more predefined reference images based on some statistical criterion. The cross correlation function is considered to be the most common criterion in such cases. [3, 4]. Such correction systems are referred to as correlation-extreme navigation systems (CENS) [1, 5]. They allow to determine the position of the vehicle by comparing the received results with the data obtained beforehand and stored in the on-board computer. As a result, the route of a vehicle equipped with inertial navigation systems is corrected regardless of weather conditions. Navigation of ships may use stellar inertial systems, where the current positions of astronomical objects relative to the object of navigation are compared.

The disadvantage of the majority of the correction navigation systems is poor resistance to variations of geometric forms due to geometry of sighting of navigation information sensors, and informational characteristics of sighted objects on the image, due to changes in weather and seasonal conditions.

\subsection{Problem analysis}

The electrophysical characteristics of different materials and backgrounds defining the data fields of survey sensors are subjected to significant variations due to changing weather and seasonal conditions, humidity, precipitation during measurements and other factors. Therefore, in the context of the need for NS to operate under any conditions of use, it is necessary to have the data of signals that are invariant to the given variations in informative parameters irrespective of the type of sensor and informative parameters. Furthermore, the integration of heterogeneous information in order to improve the accuracy of navigation systems imposes even more stringent requirements for the stability of informational parameters $[1,2]$.

With this aim in view, we propose to select an optimal RI corresponding to a stable component of an informative parameter for each comparison act for the CI fragment, for 
example: brightness, for which it is proposed to use the concept of scale of measurement theory [5] that both the brightness component of the RI and the shifts and the turns of the RI relative to the CI, as well as the operation of the enumeration of the CI fragments having the size of the RI, can be formalized in terms of the Granander Image Theory, the fundamental principles of which are outlined as follows [6]. A set F of non-produced elements called generators introduces the equivalence ratio relative to which this set breaks down into classes of equivalent elements. In addition, a similarity transformation $g$ is introduced toF, the set of which G must satisfy certain requirements, namely: to have a group structure relative to the operation of the transformation composition and to display classes of equivalent elements in itself. For the problem to be solved, we are interested in the generators of a particular type, which represent a reference space $\mathrm{X}$ in a comparable space $\mathrm{Y}$ and are referred to as forming correspondents. By definition, the effect of an elementg $\epsilon$ $G$ on an element $f \in F$ is determined by the ratio $y=$ $\mathrm{f}\left(\mathrm{g}^{-1} \mathrm{x}\right)$.

In relation to the noise component of the CI, the problem is statistical and is solved based on the theory of statistical solutions by finding the extreme quality criterion (for example, the function of plausibility of the parameter of shift of a comparable fragment relative to the $\mathrm{CI}$ ).

The purpose of the article is to synthesize reference images for correction navigation systems based on the tracking and comparison method of correction signals generation.

\section{MAIN MATERIAL}

\subsection{Introduction of transformation of a semblance on a background to describe a set of twisted and shifted RI}

The RI in the mentioned systems is formed indirectly by means of the identification of the aerial photograph (AP) of the location area with the possible precision of the result when using digital maps of the terrain. Identification of AP consists of construction in some system of coordinates $\left(\mathrm{x}_{1}, \mathrm{x}_{2}\right)$, linked to the AFS, of a mapping:

$$
\mathrm{a}: \mathrm{A} \rightarrow \mathrm{O}
$$

where $A \in \mathbf{R}^{2}$ - is the compact subset of the plane describing the RI:

$\mathrm{O}$ - is the set of types of backgrounds that are identified on AP in the target area (e.g., $\mathrm{O}=\{$ water, sand, grass, concrete\}). Hereinafter, this set will be called object composition.

Thus, the display (1) compares each point $\mathbf{x}=\left(\mathrm{x}_{1}, \mathrm{x}_{2}\right) \in$ A with the type of background according to the AP identification. The different types of backgrounds may have approximately the same radiative capacity in the millimeter wave range, in such cases they must be categorized as of the same type. It is generally considered that $4 \ldots .5$ types of backgrounds are clearly defined in radiometry [1].
The RI must be represented as a number $\mathrm{M}_{1} \times \mathrm{M}_{2}$-matrix and be formed for each reference point of the calculation trajectory defined by the flight task. Hereinafter, RI will be referred to as an image formed to be compared with $\mathrm{CI}$ obtained in a single binding session. In order to synthesize

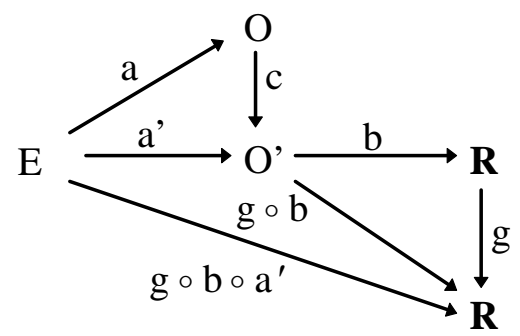

Figure 1: Chart linking the population of the introduced sets and displays

$\mathrm{RI}$, it is required to calculate the coordinates of the $\mathbf{x}^{\mathrm{ij}}=\left(\mathrm{x}_{1}^{\mathrm{ij}}, \mathrm{x}_{2}^{\mathrm{ij}}\right)\left(\mathrm{i} \in \overline{1, \mathrm{M}_{1}}, \mathrm{j} \in \overline{1, \mathrm{M}_{2}}\right)$ intersections of the axes in the antenna radiation pattern (ARP) with the background and to define the background type for each point in accordance with the AP identification. The RI can be presented both as a matrix and as a vector, by unfolding it in different ways, such as lines, which will be considered thereafter.

Let us represent the set of numbers of RI elements in its vector representation with $\mathrm{E}=\overline{1, \mathrm{M}}\left(\mathrm{M}=\mathrm{M}_{1} \mathrm{M}_{2}\right)$. It follows from the previous reasoning that a display $\mathrm{a}: \mathrm{E} \rightarrow \mathrm{O}$ is constructed, which associates the type of background with each counting number of the RI element at a point where the axis of the partial ARP with this number crosses the surface. Some elements of the RI may belong to one type of background. Thus, the display a breaks down many elements of the RI into homogeneous areas called zones.

It is possible to break down the reference set of $\mathrm{E}$ numbers into areas by introducing a binary ratio $\mathrm{I}=$ "belonging to a type of background". It can be demonstrated that the ratio I is symmetrical, reflexive and transitive, i.e. is the ratio of equivalence. It has been known [7] that the equivalence ratio divides the reference set $\mathrm{E}$ into non-cross-sectional classes of equivalents, while the set of equivalence classes(zones) is commonly referred to as the factor-set in relation to I and denoted as E/I. The displaya' $: \mathrm{E} \rightarrow \mathrm{E} / \mathrm{I}$ is referred to as a natural projection. The set $\mathrm{O}$ and $\mathrm{O}^{\prime}=\mathrm{E} / \mathrm{I}$ are simply isomorphic. The isomorphism shall be determined by an unambiguous correspondencec: $\mathrm{O} \rightarrow \mathrm{O}^{\prime}$, which associates each type of background with the corresponding class of equivalent elements. It is more convenient to work with the setO'. We denote by $\mathrm{N}$ the number of zones, and byN $\mathrm{N}_{\mathrm{i}} \subset \mathrm{E}(\mathrm{i} \in \overline{1, N})-\mathrm{a}$ set of numbers of elements that have fallen into the i-zone.

The next step is to fill in the RI according to brightness criterion, i.e. to construct a display $\mathrm{b}: \mathrm{O}^{\prime} \rightarrow \mathbf{R}$ that will match the brightness of each zone with the corresponding background type. Since the variability of the brightness of each zone makes it impossible to compare the numerical luminance unambiguously, it is proposed to use the results of the measurement theory to describe the brightness of the RI. 
Binary ratio on a set $X$ is called a subsetR $\subset X \times X$. By analogy the $k$ relation to $X$ is referred to as a subset $R$ of a

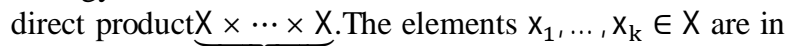
$\mathrm{k}$ times

relation to $\mathrm{R}$, if $\left(\mathrm{x}_{1}, \ldots, \mathrm{x}_{\mathrm{k}}\right) \in \mathrm{R}$.. Within the set $\mathrm{X}$ several relations can be defined, whose population $\left\{\mathrm{R}_{\mathrm{k}}\right\}_{\mathrm{k}=1}^{\mathrm{K}}$ is identified as a relation family toX. The pair $\mu=\left\langle X,\left\{R_{k}\right\}\right\rangle$ is called an empirical relation system. The main purpose of measurement theory is to assign numerical values to the elementsX. Homomorphism of the system $\mu$ into the system of numerical relations $v=\left\langle\mathbf{R},\left\{\rho_{\mathrm{k}}\right\}\right\rangle(\mathbf{R}-$ as a set of physical numbers), sometimes called a mere measurement, is referred to as the functionf: $X \rightarrow \mathbf{R}$, which retains the relation $R_{k}$. An ordered triple $\langle\mu, \nu, f\rangle$ is identified as a scale, and a set $\mathbf{R}$ - as the scale carrier. The introduced sets and corresponding displays are illustrated by the diagram shown in Figure 1

The majority of scales used in a real-case scenario allows a description of permissible transformations $\mathrm{g}: \mathbf{R} \rightarrow \mathbf{R}$. with the helps of a groupG. For such scales, if a group $\mathrm{G}$ is set up and some homomorphism $\mathrm{f}: \mathrm{X} \rightarrow \mathbf{R}$, is constructed, then the set $F_{G}(f)$ of all homomorphisms can be described as follows $F_{G}(f)=\{g \circ f, g \in G\}$. Set $F_{G}(f)$ are called the $g$ orbit of the elementf $\in \mathrm{F}$, whereF- is the set of all functions $X \rightarrow \mathbf{R}$. If subjected to the action of the group $G$, the set $\mathrm{F}$ decays into non-disposed orbits (classes), each of which corresponds to a particular relation system. Consequently, the group $\mathrm{G}$ unambiguously determines the type of scale (nominal, sequential, etc.) and vice versa. In other words, isomorphism has been established between a set of permissible transformation groups (PTG) orbits and a set of systems with relations [8].

Table 1 shows the most frequently used PTG and the corresponding scale types encountered in measurement theory.

Table 1: The population of the scales used in a real-case scenario and PTG, generating them

\begin{tabular}{|l|l|l|}
\hline Type of scale & \multicolumn{1}{|c|}{$\begin{array}{c}\text { Permissible transformation } \\
\text { group }\end{array}$} & $\begin{array}{l}\text { The } \\
\text { carrier }\end{array}$ \\
\hline Absolute & $\begin{array}{l}\text { Accurate transformation } \\
\mathrm{g}(\mathbf{x})=\mathbf{x}\end{array}$ & $\mathbf{R}$ \\
\hline Relations & $\begin{array}{l}\text { Transformation of a semblance } \\
\mathrm{g}(\mathbf{x})=\alpha \mathbf{x}, \alpha>0\end{array}$ & $(0, \infty)$ \\
\hline difference & $\begin{array}{l}\text { shift transformation } \\
\mathrm{g}(\mathbf{x})=\mathbf{x}+\mathbf{1} \beta, \beta \in \mathbf{R}\end{array}$ & $\mathbf{R}$ \\
\hline intervals & $\begin{array}{l}\text { linear transformation } \\
g(\mathbf{x})=\alpha \mathbf{x}+\mathbf{1} \beta, \\
\beta \in \mathbf{R}, \alpha>0\end{array}$ & $\mathbf{R}$ \\
\hline $\begin{array}{l}\text { Hyperordinal } \\
\text { number }\end{array}$ & Hypermonotone transformation & $\mathbf{R}$ \\
\hline $\begin{array}{l}\text { ordinal } \\
\text { number }\end{array}$ & strictly increasing generation $\mathbf{R}$ & $\mathbf{R}$ \\
\hline $\begin{array}{l}\text { Noun } \\
\text { (nominal) }\end{array}$ & Unambiguous generation $\mathbf{R}$ & $\mathbf{R}$ \\
\hline
\end{tabular}

Let us use the formalism of the Granander Image Theory to describe RI. As a reference space, we will take a set $\mathrm{X}=\left\{\mathbf{x}^{\mathrm{ij}}\right\}$, and the functionf, which displays a background space $\mathrm{X}$ in the context of a comparable space $\mathrm{Y}=\mathbf{R}$, as a generator, and by $\mathrm{F}$ a set of all such functions will be identified. Thus, the generator is a semitotonic image and its values describe the brightness of the image. Previously, these generators were identified as generatorscorrespondences. For them, the similarities can be introduced both on the background and on the comparable space. Conversions of the similarity at the comparable space describe the transformations of the brightness of the image, while similarity transformation groups Gand the corresponding scale types are shown in table 1.

The scale type is determined experimentally by multiple measurements of the brightness of each type of background surface under different conditions. Under the action of the group $\mathrm{G}$ the set $\mathrm{F}$ breaks down into orbits, each of which concentrates functions that retain certain relations on the brightness of the elements of the RI and, therefore, are similar. Such functions are referred to as digitization [9].

As a set of semblance transformations on the background space $\mathrm{X}$ we enter a set $\mathrm{S}=\mathrm{P} \times \mathrm{O}_{2}$, where $\mathrm{O}_{2}$ is a group of rotations of the Euclidean plane $\mathbf{R}^{2}$,

Pis a subset of a group of shifts $\mathrm{S}_{\mathrm{c}}$ within the half-width of the RI sampling interval.

If denoted with $\Delta \mathrm{L}_{1}, \Delta \mathrm{L}_{2}$ an average distance by points $\mathbf{x}^{\mathrm{ij}}$ at an corresponding coordinate, it is possible to determine $\mathrm{P}=\left\{\boldsymbol{\rho} \in \mathrm{S}_{\mathrm{c}}|| \rho_{1}\left|<\Delta \mathrm{L}_{1},\right| \rho_{2} \mid<\Delta \mathrm{L}_{2}\right\}$.

The element $\mathrm{O}_{\beta}$ of the rotational group $\mathrm{O}_{2}$ is the rotation of the plane by the angle $\beta$ of the counter-clockwise arrow and is described by the orthogonal matrix:

$$
\mathrm{O}_{\beta}=\left[\begin{array}{cc}
\cos \beta & -\sin \beta \\
\sin \beta & \cos \beta
\end{array}\right] \text {. }
$$

The action of an element $\mathrm{s}=\left(\boldsymbol{\rho}, \mathrm{O}_{\beta}\right) \in \mathrm{S}$ in space $\mathrm{X}$ is described by the expression:

$$
\mathbf{r}=\mathrm{s} \mathbf{x}=\boldsymbol{\rho}+\mathrm{O}_{\beta} \mathbf{x}
$$

or in the coordinates

$$
\begin{aligned}
& r_{1}=\rho_{1}+x_{1} \cos \beta-x_{2} \sin \beta \\
& r_{2}=\rho_{2}+x_{1} \sin \beta+x_{2} \cos \beta .
\end{aligned}
$$

We determine the transformation $s \in S$ action on the set of generators $\mathrm{F}$ as follows:

$$
\mathrm{f}(\mathrm{s} \mathbf{x})=\mathrm{f}\left(\boldsymbol{\rho}+\mathrm{O}_{\beta} \mathbf{x}\right)
$$

Thus, the introduction of semblance transformations on the background space makes it possible to describe a set of shifted and rotated RI, so that, when compared with the CI fragment, the most suitable RI for the comparable fragment can be obtained.

\subsection{Current image model}

Suppose a scalar brightness field $\psi(\mathbf{y})=\psi\left(\mathrm{y}_{1}, \mathrm{y}_{2}\right)$. is set on the sighting surface in some coordinate system $\left(\mathrm{y}_{1}, \mathrm{y}_{2}\right)$.

In CENS, the field is discredited and smoothed out by a spatial filter: 


$$
\mathrm{p}_{\mathrm{ij}}=\int_{\mathbf{R}^{2}} \mathrm{~L}(\mathbf{y}) \psi(\mathbf{y}) \mathrm{H}\left(\mathbf{y}^{\mathrm{ij}}-\mathbf{y}\right) \mathrm{d} \mathbf{y}, \mathrm{i} \in \overline{1, \mathrm{~N}_{2}}, \mathrm{j} \in \overline{1, \mathrm{~N}_{1}}(5)
$$

where $\mathrm{L}(\mathbf{y})-$ is the coefficient, which accounts for propagation path attenuation of the signal;

$\mathrm{H}(\mathbf{y})$ - is the function of the antenna radiation pattern converted in relation to the spatial coordinates on the background;

$\mathbf{y}^{\mathrm{ij}}-$ is the intersection of the axis of the $(i, j)$ direction chart with the sighting surface;

$\mathrm{N}_{1}, \mathrm{~N}_{2}-$ are the dimensions of the CI matrix.

The operation of enumeration of CI fragments having the matching dimensions of the RI is convenient to formalize in terms of image theory. We introduce as a supporting space a subset $\mathrm{Y}_{0}=\overline{1, \mathrm{~N}_{1}} \times \overline{1, \mathrm{~N}_{2}}$ of integer lattice $\mathbf{Z}^{2}$ (where $\mathbf{Z}$ is a set of whole numbers).

Then CI is the function $\mathrm{p}^{\prime}: \mathrm{Y}_{0} \rightarrow \mathbf{R}$.. The generator can be defined as the reduction of this function by a subset $\mathrm{Y}=$ $\overline{1, \mathrm{M}_{1}} \times \overline{1, \mathrm{M}_{2}}$, i.e.p $=\left.\mathrm{p}^{\prime}\right|_{\mathrm{Y}}$

As a set of transformation of the conformity of background space, let us select a group of integer shifts, the action of which on the base space is defined by the rule:

$$
\mathbf{q}(\mathbf{y})=\mathbf{y}+\mathbf{q}, \mathbf{y}, \mathbf{q} \in \mathbf{Z}^{2},
$$

and on the generator - by the following relation:

$$
\mathbf{q p}(\mathbf{y})=\left.\mathrm{p}^{\prime}(\mathbf{y}+\mathbf{q})\right|_{\mathrm{Y}} \cdot \mathrm{D}
$$

Consequently, the generator is a CI fragment having the matching dimensions of the RI, and the application of the integer shift operation to the generator results in the formation of new CI fragments with the same dimensions. The set $\mathrm{Q}$ of the permissible shifts where the selected fragments do not exceed the limits of the CI, is equal toQ $=\overline{0, N_{1}-M_{1}} \times \overline{0, N_{2}-M_{2}}$.

Suppose that CI is accepted against the background of additive noisen', whilst the noise in individual channels is independent and distributed according to the standard law, i.e.:

$$
\mathrm{I}^{\prime}=\mathrm{p}^{\prime}+\mathrm{n}^{\prime}
$$

where $\mathrm{p}^{\prime}-$ is noise-free $\mathrm{CI}$;

$\mathrm{n}^{\prime}: \mathrm{Y}_{0} \rightarrow \mathbf{R}-$ is a random function that describes the system channels interior noises.

Let the function $\mathrm{D}: \mathrm{Y}_{0} \rightarrow \mathbf{R}$ describe the noise dispersion of the system channels.

After converting in the expression (8) to the generator the following is obtained:

$$
\mathrm{I}=\mathrm{p}+\mathrm{n}
$$

where $\mathrm{I}, \mathrm{n}-$ of the contraction $\mathrm{I}^{\prime}, \mathrm{n}^{\prime}$ Yat.

It is customary to call the source fragment a fragment that actually corresponds to the RI.

Let $\mathbf{q}_{0}$ is the shift that matches the selection of this fragment.

Then

$$
\left.\mathbf{q}_{\mathrm{o}} \mathrm{p}^{\prime}\left(\mathrm{y}_{1}, \mathrm{y}_{2}\right)\right|_{\mathrm{Y}}=\operatorname{sgf}\left(\mathrm{x}_{1}, \mathrm{x}_{2}\right)
$$

i.e. the source fragment corresponds to some RI digitizationf, and this digitization should be chosen in such a way that it best corresponds to the source fragment, which is achieved by choosing the appropriate transformation of the similarityg $\in \mathrm{G}$, and to compensate for the displacement and rotation of the CI coordinate system in relation to the RI coordinate system, an element $\mathrm{s} \in \mathrm{S}$ has to be selected.

Let us include (9) to the coordinates with regard to (4):

$$
\begin{aligned}
& I^{\prime}\left(i+q_{1}, j+q_{2}\right)=g f\left(\rho_{1}+x_{1}^{i j} \cos \beta-x_{2}^{i j} \sin \beta, \rho_{2}+x_{1}^{i j} \sin \beta+x_{2}^{i j} \cos \beta\right)+ \\
& +n^{\prime}\left(i+q_{1}, j+q_{2}\right),(i, j) \in Y .
\end{aligned}
$$

\subsection{The formalization of the RI synthesis task based on the statistical solution theory}

From the point of view of statistical solution theory, the task of finding the CI fragment, which is the most "similar" to the RI, based on the additive model (9) of the interaction of the image with noise is formulated as follows: the shift $\mathbf{q}$ is to be evaluated; this shift provides the extremum to the chosen quality criterion, if there are nuisance parameterss, g, which should also be assessed in the process of the task solution.

As a quality criterion, we select the logarithm of the function of probability of the parameters $\mathbf{q}, \mathrm{s}, \mathrm{g}$, based on (11), can be calculated, with precision to the points which do not affect the position of the maximum value of the parameters, by the expression:

$\Lambda(\mathbf{q}, \mathrm{s}, \mathrm{g})$
$=-\sum_{\substack{\mathrm{i}=1 \\ \mathrm{M}_{1}}}^{\mathrm{M}_{\mathrm{j}}} \sum_{\mathrm{j}=1}^{\mathrm{M}_{2}} \ln \left[\mathrm{D} \Theta\left(\mathrm{i}+\mathrm{q}_{1}, \mathrm{j}+\mathrm{q}_{2}\right)\right]$
$-\sum_{\mathrm{j}=1}^{\mathrm{M}_{2}} \frac{\left[\mathrm{I}^{\prime}\left(\mathrm{i}+\mathrm{q}_{1}, \mathrm{j}+\mathrm{q}_{2}\right)-\operatorname{gf}\left(\rho_{1}+\mathrm{x}_{1}^{\mathrm{ij}} \cos \beta-\mathrm{x}_{2}^{\mathrm{ij}} \sin \beta, \rho_{2}+\mathrm{x}_{1}^{\mathrm{ij}} \sin \beta+\mathrm{x}_{2}^{\mathrm{ij}} \cos \beta\right)\right]^{i}}{\mathrm{D}^{\prime}\left(\mathrm{i}+\mathrm{q}_{1}, \mathrm{j}+\mathrm{q}_{2}\right)}$

Let us expand the RI and the CI fragments by lines, starting from the bottom line Then RI is displayed as a vectore $==\left(\mathrm{e}_{1}, \ldots \mathrm{e}_{\mathrm{M}}\right) \in \mathbf{R}^{\mathrm{M}}$, where

$$
\mathrm{e}_{\mathrm{M}_{1}(\mathrm{j}-1)+\mathrm{i}}=\mathrm{f}\left(\mathbf{x}^{\mathrm{ij}}\right),(\mathrm{i}, \mathrm{j}) \in \mathrm{Y} .
$$

For CI, the fragment number, corresponding to the vector shift $\mathbf{q}$, is defined by the expression:

$$
\mathrm{k}=\left(\mathrm{N}_{1}-\mathrm{M}_{1}+1\right) \mathrm{q}_{2}+\mathrm{q}_{1}+1, \mathbf{q} \in \mathrm{Q} .
$$

The shift vector $\mathbf{q}$ is regenerated by the number $\mathrm{k}$ with the help of the relation:

$$
\mathbf{q}=\left(\bmod _{\mathrm{N}_{1}-\mathrm{M}_{1}+1} \mathrm{k}-1, \operatorname{div}_{\mathrm{N}_{1}-\mathrm{M}_{1}+1} \mathrm{k}\right)
$$

The $\mathrm{k}$ fragment of CI and noise are described by vectors $\mathbf{y}^{\mathrm{k}}=\left(\mathrm{y}_{1}^{\mathrm{k}}, \ldots, \mathrm{y}_{\mathrm{M}}^{\mathrm{k}}\right), \mathbf{d}^{\mathrm{k}}=\left(\mathrm{d}_{1}^{\mathrm{k}}, \ldots, \mathrm{d}_{\mathrm{M}}^{\mathrm{k}}\right)$, whose components 
are equal to the values of the function $\mathrm{I}^{\prime}$ and to the reciprocal values the function $\mathrm{D}^{\prime}$ respectively.

Then (12) can be presented as:

$$
\Lambda(\mathrm{k}, \mathrm{s}, \mathrm{g})=-\mathrm{L}(\mathrm{k})-\mathrm{B}(\mathrm{k}, \mathrm{s}, \mathrm{g}),
$$

where

$$
\mathrm{L}(\mathrm{k})=\sum_{\mathrm{i}=1}^{\mathrm{M}} \ln \left(\mathrm{d}_{\mathrm{i}}^{\mathrm{k}}\right), \mathrm{B}(\mathrm{k}, \mathrm{s}, \mathrm{g})=\sum_{\mathrm{i}=1}^{\mathrm{M}} \mathrm{d}_{\mathrm{i}}^{\mathrm{k}}\left(\mathrm{y}_{\mathrm{i}}^{\mathrm{k}}-\mathrm{ge}_{\mathrm{i}}^{\mathrm{s}}\right)^{2}
$$

\subsection{The synthesis of an optimal algorithm for generating the correction signal}

To synthesize an optimal algorithm the problem has to be solved:

$$
(\widehat{\mathbf{q}}, \hat{\mathrm{s}}, \hat{\mathrm{g}})=\arg \max _{\mathrm{q} \in \mathrm{Q}, \mathrm{s} \in \mathrm{S}, \mathrm{g} \in \mathrm{G}} \Lambda(\mathrm{k}, \mathrm{s}, \mathrm{g}),
$$

which is equivalent to the task

$$
(\hat{\mathrm{k}}, \hat{\mathrm{s}}, \hat{\mathrm{g}})=\arg \min _{\mathrm{k} \in \mathrm{K}, \mathrm{s} \in \mathrm{S}, \mathrm{g} \in \mathrm{G}}-\Lambda(\mathrm{k}, \mathrm{s}, \mathrm{g}),
$$

if you expand the eigenvalue matrix of criterial function $\Lambda$ by lines. In formula (18) $\mathrm{K}=\left(\mathrm{N}_{1}-\mathrm{M}_{1}+1\right)\left(\mathrm{N}_{2}-\mathrm{M}_{2}+\right.$ $+1)$ is the number of $\mathrm{CI}$ fragments.

Since the first part of (16) doesn't depend ofs, g,, the task must first be solved:

$$
(\hat{s}, \hat{g})=\arg \min _{s \in S g \in G} B(k, s, g),
$$

and then it is necessary to find the number $\hat{k}$, for which

$$
\mathrm{L}(\hat{\mathrm{k}})+\mathrm{B}(\hat{\mathrm{k}}, \hat{\mathrm{s}}, \hat{\mathrm{g}})<L(k)+B(k, \hat{\mathrm{s}}, \hat{\mathrm{g}}), \mathrm{k} \in \overline{1, \mathrm{~K}}, \mathrm{k} \neq \hat{\mathrm{k}}
$$

The task (19) is the selection of a "reference of maximum similarity" for this particular CI fragment according to V.A.Kovalevsky's terminology [10].

In practice, it is useful to select a discrete parameters, i.e. to construct an RI set $\left\{\mathrm{e}^{\mathrm{s}}\right\}_{\mathrm{s} \in \mathrm{S}}$, each element of which is characterized by a shift vector $\rho$ and a rotation angle $\beta$.

To achieve the objective (19), it is first necessary to:

$$
\mathrm{g}^{\wedge}=\underset{\mathrm{g} \hat{\mathrm{I} G}}{\operatorname{argmin} B}(\mathrm{k}, \mathrm{s}, \mathrm{g})
$$

It is convenient to present $\mathrm{B}(\mathrm{k}, \mathrm{s}, \mathrm{g})$ as the form of a norm in a bilinear-metric space with corresponding metric.

Lowering fixed indexes $\mathrm{k}, \mathrm{s}$, to an accuracy independent of the $g$ multiplier leads (20) to the following appearance:

$$
\mathrm{g}^{\wedge}=\underset{\mathrm{g} \hat{\mathrm{I} G}}{\operatorname{argmin}} \sum_{\mathrm{i}=1}^{\mathrm{M}} \mathrm{p}_{\mathrm{i}}\left(\mathrm{y}_{\mathrm{i}}-\mathrm{gz}_{\mathrm{i}}\right)^{2},
$$

where

$$
\mathrm{p}_{\mathrm{i}}=\mathrm{d}_{\mathrm{i}} / \sum_{\mathrm{i}=1}^{\mathrm{M}} \mathrm{d}_{\mathrm{i}}
$$

It follows from expression $(22)$ that $\sum \mathrm{p}_{\mathrm{i}}=1$. Furthermore, as $d_{i}-$ is the value, which is opposite to the noise dispersion of $\mathbf{i}$ channel. Thus, the population of weight coefficients $\left\{\mathrm{p}_{\mathrm{i}}\right\}$ determines a bilinear symmetrical positively, by the particular shape with a diagonal matrix $A=\operatorname{diag}\left(\mathrm{p}_{1}, \ldots, \mathrm{p}_{\mathrm{M}}\right)$, which produces in $\mathrm{R}^{\mathrm{M}}$ metric, in regards to which the problem (11) can be recorded as:

$$
\mathrm{g}^{\wedge}=\underset{\mathrm{g} \in \mathrm{G}}{\operatorname{argmin}}\|y-\mathrm{g} z\|_{A}^{2}
$$

The task of the definition of the optimal transformation of the RI from the PTG in the case of the first four scales, stated in the Table 1, which are defined as strong, is convenient to solve in the production described by the expression (23). For other types of scales called weak, it is useful to use the RI area structure, which allows to reduce the size of the task.

If the PGT G is found and the homomorphism $\mathrm{f}: \mathrm{X} \rightarrow \mathrm{R}$ of the empirical $\mu=(X, R)$ (and numerical $\nu=\langle\mathbf{R}, \rho\rangle$ scales is determined, the relation $\mathbf{R} \subset \mathbf{X}^{2}$ is set on the reference background $\mathrm{X}$ (in case of the order scale it is the relation of the linear quasi-order, in case of the scale of the hyperorder it is hyperorder relation, in case of the nominal scale it is the relation of equivalence).

IfR- is the relation of a linear quasi-order, it can be represented as the sum of a strict relation $\mathrm{R}^{\mathrm{S}}$ and a relation of equivalence $\mathrm{R}^{\mathrm{I}}$ [8]:

$$
\mathrm{R}=\mathrm{R}^{\mathrm{s}}+\mathrm{R}^{\mathrm{I}} \text {. }
$$

A relation $R^{I}$ divides space $X$ into zones, and a relation $R^{s}$ establishes a relation of strict order in multiple zones $\mathrm{N}$. Since the seNt is now ordered, lets renumber its elements in the order of their brightness. Lets include in (11) $\boldsymbol{\kappa}=$ gzand reformulate the restriction $g \in G$ into the limit for vectork.

We will construct the display $r: X \rightarrow \overline{1, N}$, that compares the $\mathbf{i}$ RI element to the zone $\mathrm{j}$ number, to which it belongs.

The vector $\mathbf{r}=\left(\mathrm{r}_{1}, \ldots, \mathrm{r}_{\mathrm{M}}\right)$ is called a vector of the rank elements of the RI.

Let us build a line operatorh: $\mathrm{R}^{\mathrm{N}} \rightarrow \mathrm{R}^{\mathrm{M}}$, which hasM $\times \mathrm{N}$, which is the matrixHwith elements of $h_{i j}=\delta_{r_{i} j}\left(\delta_{i j}-\right.$ the symbol of Kroeker).

In the matrix $\mathbf{H}$ in $\mathrm{i}$-line in $\mathrm{j}$-field there is 1 if $\mathrm{i} \mathrm{RI}$ element belongs to $\mathrm{j}$-zone and the rest of the line elements are equal to zero. We introduce a vector $\boldsymbol{\pi} \in \mathbf{R}^{\mathrm{N}}$, whose components are equal to digitization of the brightness of zones. Then any RI digitization vector $\boldsymbol{\kappa} \in \mathbf{R}^{\mathrm{M}}$ can be expressed through the vector $\boldsymbol{\pi}$ by the relation:

$$
\kappa=\mathbf{H} \boldsymbol{\pi} .
$$

Lets characterized through $\mathrm{C}_{\boldsymbol{\pi}} \subset \mathbf{R}^{\mathrm{N}}$ the set of permissible digitizations by area, which is determined by the relation $\rho$ in the numerical scale. Then, the set of permissible digitizations for the vector $\boldsymbol{\kappa} \in \mathbf{R}^{\mathrm{M}}$ can be described as follows:

$$
\mathrm{C}_{\boldsymbol{\kappa}}=\left\{\boldsymbol{\kappa} \in \mathbf{R}^{\mathrm{M}} \mid \boldsymbol{\kappa}=\mathbf{H} \boldsymbol{\pi}, \boldsymbol{\pi} \in \mathrm{C}_{\boldsymbol{\pi}}\right\}
$$

The task (23) in the case of representation of RI in weak scales takes the form of: 


$$
\widehat{\boldsymbol{\kappa}}=\underset{\mathbf{\kappa} \in \mathbf{C}_{\mathbf{\kappa}}}{\operatorname{argmin}}\|\mathbf{y}-\mathbf{\kappa}\|_{\mathbf{A}}^{2}
$$

It can be shown that the task (25) is equivalent to the following:

$$
\widehat{\boldsymbol{\pi}}=\underset{\pi \in C_{\boldsymbol{\pi}}}{\operatorname{argmin}}\|\mathrm{y}-\mathbf{H} \boldsymbol{\pi}\|_{\mathrm{A}}^{2}
$$

The optimum digitization $\widehat{\boldsymbol{\kappa}}$ is restored along the vector $\widehat{\boldsymbol{\pi}}$ by means of a relation (24).

\section{CONCLUSION}

As a result of the conducted research, the optimal general RI and algorithms for vehicle navigation systems, invariant to the CI linear transformation group, are synthesized without reference to a specific sensor type and informative parameter.

The generalized relations for describing the models of current and reference images and optimum algorithms for forming corrective signals for the main navigation system have been obtained.

\section{REFERENCES}

1.V. Antyufeev. Matrix radiometric correlationextreme navigation systems for aircraft: monographUkraine, Kharkov: KhNU V.N. Karazin, 372 p.

2.A. Sotnikov, V. Tarshyn, N. Yeromina, S. Petrov, N. Antonenko. A method for localizing a referenceobject in a current image with several bright objects, EasternEuropean Journal of Enterprise Technologies. 2017. Vol. № 9 (87). pp. 68 - 74. DOI: $10.15587 / 1729$ 4061.2017.101920.

3.N.Yeromina, S. Petrov, A. Tantsiura, M. Iasechko, V. Larin. Formation of reference images and decisionfunction in radiometric correlation-extremal navigation systems. Eastern-European Journal ofEnterprise Technologies. - 2018. Vol.4, No.9 (94). - pp. 27 35. DOI: $10.15587 / 1729-4061.2018 .139723$

4. O. Vorobiov, A. Sotnikov, A Tantsiura. Models ofcurrent images that are formed by the combined correlation-extremal navigation system of a nonflammable aircraft. Modern information technologiesin the sphere of security and defence. Ukraine, Kyiv, - 2018. Vol.2, No.32. - pp. 29 - 36.

5. Pfanzagl I. Measurement theory. - Moscow: Mir, 1976. $-248 \mathrm{p}$.

6. Grenander W. Lectures on the theory of images : TRANS.: In 3 t. - M.: Mir. Vol. 1: image Synthesis. 1979. $-383 \mathrm{p}$.

7. Mirkin B. G. Analysis of qualitative features. Moscow: Statistics, 1976. - $166 \mathrm{p}$.

8. Kuzmin V. B. Construction of group solutions in spaces of clear and nonclear binary relations. Moscow: Nauka, 1982. - 168 p.

9. Data analysis methods: an Approach based on the dynamic thickening method. - Moscow: Finance and statistics, 1985. - $359 \mathrm{p}$.

10. Kovalevsky V. A. Methods of optimal solutions in image recognition. - Moscow: Nauka, 1976. - 328 p. 